Artículo

\title{
Carbono en suelo, hierbas y arbustos en una plantación forestal en Jalisco, México
}

\author{
Miguel Acosta Mireles ${ }^{1}$ \\ Fernando Carrillo Anzures ${ }^{1 \S}$ \\ Enrique Buendía Rodriguez ${ }^{1}$ \\ Juan de Dios Benavides Solorio ${ }^{2}$ \\ Eulogio Flores Ayala ${ }^{1}$ \\ Lucila González Molina ${ }^{1}$ \\ ${ }^{1}$ Campo Experimental Valle de México-INIFAP. Carretera Los Reyes-Texcoco km 13.5, Coatlinchán, \\ Texcoco, Estado de México, México. CP. 56250. (acosta.miguel@inifap.gob.mx; \\ buendia.enrique@inifap.gob.mx; flores.eulogio@inifap.gob.mx; gonzalez.lucila@inifap.gob.mx). ${ }^{2}$ Campo \\ Experimental Centro Altos de Jalisco-INIFAP. Parque Los Colomos s/n, Colonia Providencia, Guadalajara, \\ Jalisco. CP. 44660. (benavides.juan@inifap.gob.mx). \\ ${ }^{\S}$ Autor para correspondencia: carrillo.fernando@inifap.gob.mx.
}

\section{Resumen}

Un adecuado manejo forestal sustentable, ya sea en una plantación o un bosque natural, se crean sumideros de carbono que suelen ser constante en el tiempo, sin embargo, se desconoce cómo afecta las actividades del manejo de la vegetación en los sumideros de carbono en los ecosistemas forestales. El objetivo fue estimar la concentración de carbono en herbáceas y arbustos, hojarasca y dos profundidades de suelo ( 0 a $15 \mathrm{~cm}$ y de 15 a $30 \mathrm{~cm}$ ), en una plantación forestal con presencia de cuatro especies tropicales en dos condiciones de manejo. Se realizaron las mediciones de carbono en hierbas y arbustos, hojarasca y dos profundidades de suelo en dos condiciones con manejo y sin manejo en 30 parcelas con presencia de rosa morada (Tabebuia rosea), teca (Tectona grandis), melina (Gmelina arborea) y parota (Enterolobium cyclocarpum). Después de la colecta, se pesó y se determinó el peso seco de cada muestra, para determinar el contenido de carbono por cada uno de los componentes. Después se realizó un Anova y la prueba de Duncan para determinar si existen diferencias significativas. La parcela con parota y con manejo fue la que mayor cantidad de carbono total registró con $73.94 \mathrm{Mg} \mathrm{ha}^{-1}$, mientras que la parcela con teca con manejo reportó el menor contenido de carbono total, con $45.63 \mathrm{Mg} \mathrm{ha}^{-1}$. En cuanto al contenido de carbono en el suelo este disminuye en $35 \%$ aproximadamente, conforme aumenta su profundidad, existiendo diferencias significativas.

Palabras claves: biomasa, manejo de plantaciones, sitios de muestreo.

Recibido: mayo de 2020

Aceptado: agosto de 2020 


\section{Introducción}

En la actualidad, las consecuencias del cambio climático debido principalmente al incremento en la atmósfera de los gases efecto invernadero (GEI) tienen serios efectos negativos y si bien los escenarios futuros todavía son inciertos, es de esperarse una extinción masiva de especies, escases de agua y baja producción en cultivos agrícolas, por lo que es primordial desarrollar medidas para reducir las emisiones de los GEI. Una de las alternativas evaluadas es incrementar la captura de C en la biomasa y en los suelos mediante la preservación de los hábitats forestales existente e incrementar las plantaciones forestales como una opción de almacenes de carbono. Otra alternativa, es la realización de prácticas agrícolas adecuadas para limitar en mayor medida las emisiones de los GEI, además debe de ser implementadas en terrenos adecuados para su realización. Por lo tanto, en el futuro deben desarrollarse diferentes estrategias y políticas apropiadas para el manejo los recursos forestales y las actividades agrícolas (IPCC, 2007).

En general, los ecosistemas forestales son buenos secuestradores de $\mathrm{CO}_{2}$ atmosférico y por lo tanto son importantes almacenes del carbono, tanto en sus etapas tempranas como en las etapas tardías (bosques maduros) y en particular, las especies de rápido desarrollo puesto que se incrementa rápidamente la captura de carbono que después se convierten en depósitos de carbono. Al realizar un adecuado manejo forestal sustentable, ya sea en una plantación o un bosque natural, se crean sumideros de carbono que suelen ser constante en el tiempo, puesto que se renueva en sucesivas rotaciones los volúmenes de madera y se reincorporan mediante la realización de reforestación en la superficie forestal.

La madera proveniente de un árbol muerto se descompone por actividad bacteriana, hongos e invertebrados, los cuales reciclan el carbono como biomasa, materia orgánica muerta y una vez más regresan a la atmosfera en forma de gases $\left(\mathrm{CO}_{2}\right.$ y $\left.\mathrm{CH}_{4}\right)$ o en líquidos, que buena parte son incorporados al suelo durante los procesos de descomposición (FAO, 2000). También, otros fenómenos físicos o biológicos pueden alterar los sumideros de carbono como son el arrastre y depósito de sedimentos del suelo por erosión hídrica o eólica, la agregación de $\mathrm{CO}_{2}$ por precipitación pluvial, el traslado de seres vivos y transporte de sustancias orgánicas, pueden modificar el balance de carbono.

En México, el manejo forestal sustentable de áreas forestales ya sea de bosques naturales, zonas de reforestación, áreas naturales protegidas, o plantaciones comerciales son una de las mejores opciones para la captación de carbono, ofreciendo una alternativa para incrementar la producción maderable y no maderable de manera simultánea. Además, con estas actividades se contribuye al establecimiento de bancos de germoplasma, conservación de suelos y mantener la biodiversidad de los ecosistemas forestales (Masera et al., 2000).

$\mathrm{Al}$ respecto, existen varios estudios planteados para evaluar los reservorios de carbono en el suelo, entre los cuales se hallan los trabajos realizados en el bosque tropical seco (Gómez, 2008), en el sureste de México (De Jong, 1995; De Jong et al., 1999; Soto et al., 2010), en la Sierra Norte de Oaxaca (Acosta et al., 2002) y en el centro occidente (Ordóñez et al., 2001). Sin embargo, se desconoce cómo afecta las actividades del manejo de la vegetación en los sumideros de carbono en los ecosistemas forestales (Figueroa et al., 2005). 
El objetivo fue estimar la concentración de carbono en herbáceas y arbustos, hojarasca y dos profundidades de suelo (0 a $15 \mathrm{~cm}$ y de 15 a $30 \mathrm{~cm}$ ), en una plantación forestal con presencia de cuatro especies tropicales en dos condiciones de manejo, establecidas en el Sitio Experimental Costa de Jalisco del Instituto Nacional de Investigaciones Forestales Agrícolas y Pecuarias (INIFAP) en el estado de Jalisco.

\section{Materiales y métodos}

\section{Área de estudio}

La zona de estudio se ubica dentro del Sitio Experimental Costa de Jalisco, perteneciente al INIFAP, localizado entre los municipios de la Huerta y Casimiro Castillo en el Estado de Jalisco, entre las coordenadas $19^{\circ} 31^{\prime} 15^{\prime}$ "de latitud norte y $104^{\circ} 32^{\prime}$ '00" de longitud oeste, a una altitud promedio de $298 \mathrm{msnm}$. El clima es AW2 (w) tipo cálido subhúmedo con lluvias en verano y con una precipitación media anual de 1100 mm., según la clasificación de Köppen modificado por García (2004).

\section{Toma de datos}

La información de campo fue recolectada en 30 parcelas provienen de una plantación con cuatro especies forestales: Tabebuia rosea (Bertol.) DC. (rosa morada), Tectona grandis L.f. (teca), Gmelina arbórea Roxb. ex Sm. (melina) y Enterolobium cyclocarpum (Jacq.) Griseb. (parota), realizada a marco real con una distancia de 4 x $4 \mathrm{~m}$ de separación entre árboles, la cual fue establecida en 1994.

El muestreo fue realizado completamente al azar en dos escenarios de manejo. Por un lado, las parcelas con manejo (eliminación de herbáceas y arbustos), donde se evaluó tres condiciones con presencia de las siguientes especies: rosa morada, teca, melina, estableciendo seis sitios de muestreo para cada parcela. Por otro lado, las parcela sin manejo donde si existe la presencia de herbáceas y arbustos, de las cuales se obtuvieron datos de biomasa de ambos componentes (herbácea y arbustos) como uno solo, en esta parcela también se establecieron 6 sitios de muestreo para cada parcela con presencia de rosa morada y parota.

Para la evaluación del contenido de carbono se utilizó una modificación de la metodología propuesta por Márquez (1997), para estimar el carbono y evaluar las variables de interés principal, en este caso dos componentes: aéreo (vegetación arbustiva, herbácea, hojarasca) y subterráneo (suelo a dos profundidades).

\section{Cuantificación de carbono en arbustos y herbáceas}

Para determinar el contenido de carbono, se establecieron seis puntos de muestreo al azar para cada parcela, utilizando como unidad de muestreo sitios cuadrados de 1 x $1 \mathrm{~m}\left(1 \mathrm{~m}^{2}\right)$. Todo el material vegetal perteneciente a los arbustos y hierbas dentro del cuadrante fue obtenido cortando toda la biomasa existente al nivel de suelo. De las plantas que estaban parcialmente fuera del sitio de muestreo, solo se consideraron las ramas o tallos que entraban en el cuadrante (Acosta et al., 2009). 


\section{Cuantificación de carbono en hojarasca}

Los sitios de muestreo establecidos para hierbas y arbustos también se utilizaron para medir la cantidad de hojarasca; sin embargo, en este caso los cuadrantes fueron de $0.25 \mathrm{~m}^{2}$ (cuadros de 50 x $50 \mathrm{~cm}$ ). En esa área se colectó la biomasa perteneciente sólo a la hojarasca o mantillo. Una vez colectada todo el material de hierbas y arbustos, y hojarasca se colocó en una bolsa de manta por separado para enviarlos al laboratorio de suelo del INIFAP en el Campo Experimental Tecomán, Colima. Donde fueron secadas en una estufa a $75^{\circ} \mathrm{C}$ durante $48 \mathrm{~h}$ para obtener el peso seco. Para convertir los datos de biomasa a carbono, se multiplicó el total de biomasa por hectárea obtenida por la constante de 0.5 , puesto que, en la mayoría de las especies la mitad de la biomasa es carbono en cualquiera de sus partes (Masera et al., 2000).

\section{Cuantificación de carbono en suelo}

Las muestras de suelo se obtuvieron en el mismo punto de muestreo donde se realizó el muestreo de los anteriores componentes. Para ello se realizó con una barrena de $8 \mathrm{~cm}$ de diámetro (AMS Soil Sampling Equipment) y se obtuvieron dos muestras de suelo para cada nivel de profundidad (0-15 y de 15-30 cm). De esa manera, se obtuvieron doce muestras de suelo por cada parcela en los sitios de muestreo previamente establecidos. Al conocer el diámetro del cilindro utilizado y su longitud, se determinó el volumen de suelo muestreado, después al analizar el suelo y determinar el porcentaje de carbono, se hicieron las inferencias de la cantidad de carbono por unidad de superficie y de acuerdo a la densidad de las muestras de suelo.

Todas las muestras de suelo fueron colocadas individualmente en bolsas de plástico con su etiqueta respectiva y se trasladó al laboratorio, en donde se secó en estufa de secado a una temperatura de $60^{\circ} \mathrm{C}$ durante $48 \mathrm{~h}$, en forma manual se removieron las raíces, piedras y otros materiales como residuos vegetales y residuos de fauna.

Posteriormente, las muestras de suelo ya libres de raíces y otros materiales, se tamizaron con un martillo de madera hasta hacerlas pasar por una malla de $2 \mathrm{~mm}$, después de homogeneizarlas completamente se extrajo una submuestra de aproximadamente $5 \mathrm{~g}$, se secó a $105^{\circ} \mathrm{C}$ y se molió durante tres minutos en un molino tipo Speck 8000 Mixer/Mill ${ }^{\circledR}$. El análisis de carbono se determinó utilizando el método de combustión directa a $1000{ }^{\circ} \mathrm{C}$, con un analizador automático de carbono total, equipado con una unidad para procesar muestras sólidas.

El análisis para determinar el carbono en las muestras de suelo se realizó en el laboratorio de suelo del INIFAP en el Campo Experimental Tecomán, Colima. Para determinar el contenido de carbono en cantidad, fue necesario conocer la densidad aparente del suelo, para ello se realizó su cálculo, mediante la obtención del volumen total del suelo y luego el ajuste, restando el volumen ocupado por raíces, piedras o cualquier otro residuo.

\section{Análisis de la información}

Se realizaron pruebas para verificar que se cumplan los supuestos de los residuales, la normalidad con la prueba de Shapiro-Wilk y la homogeneidad de varianzas mediante la prueba de Levene, ambas con un grado de significancia $(p<0.05)$, con ayuda del software $\mathrm{R}^{\odot}$ ver 3.6. Ya que cumplieron los supuestos se realizó un análisis de varianza (Anova) de un factor (parcelas) para 
los valores de carbono en arbustos, hojarasca, suelo de 0 a 15 y 15 a $30 \mathrm{~cm}$ de profundidad, se utilizó la prueba de Duncan para determinar si existían diferencias significativas $(p<0.05)$ de las variables analizadas por parcela (Zar, 2010).

\section{Resultados}

La estadística descriptiva del muestreo realizado señala que la parcela con mayor contenido de carbono en el componente de arbustos y herbáceas es la de parota con $1.63 \pm 0.63 \mathrm{Mg} \mathrm{ha}{ }^{-1}$, en cambio las parcelas con menor cantidad (nula) fueron aquellas con manejo, ya que este componente fue removido por completo. En el componente de hojarasca, las parcelas con mayor cantidad de carbono fueron las de parota con un $6.36 \pm 5.04 \mathrm{Mg} \mathrm{ha}^{-1}$, pero tienen una gran variabilidad, y la parcela con menor cantidad de carbono fueron las de rosa morada I con manejo $\left(2.9 \pm 0.81 \mathrm{Mg} \mathrm{ha}^{-1}\right)$. El mayor contenido de carbono en suelo se encontró a la profundidad de 0 a $15 \mathrm{~cm}$, en la parcela de parota con un $37.68 \pm 5.42 \mathrm{Mg} \mathrm{ha}^{-1}$ y disminuyó hasta $14.54 \pm 4.13 \mathrm{Mg}$ $\mathrm{ha}^{-1}$ en la parcela de teca a una profundidad de 15 a $30 \mathrm{~cm}$ (Cuadro 1).

Cuadro 1. Estadísticas básicas de contenido de carbono por parcela ( $\left.\mathrm{Mg} \mathrm{ha}^{-1}\right)$.

\begin{tabular}{|c|c|c|c|c|c|}
\hline & Parcela & Media & Desviación estándar & Mínimo & Máximo \\
\hline \multirow[t]{6}{*}{ Arbustos y herbáceas } & Melina & 0 & 0 & 0 & 0 \\
\hline & Teca & 0 & 0 & 0 & 0 \\
\hline & Rosa Morada I & 0 & 0 & 0 & 0 \\
\hline & Parota & 1.63 & 0.63 & 0.79 & 2.44 \\
\hline & Rosa Morada II & 1.42 & 0.63 & 0.78 & 2.2 \\
\hline & Total & 0.61 & 0.85 & 0 & 2.44 \\
\hline \multirow[t]{6}{*}{ Hojarasca } & Melina & 5.78 & 4.8 & 3.27 & 15.48 \\
\hline & Teca & 3.06 & 0.48 & 2.63 & 3.98 \\
\hline & Rosa Morada I & 2.89 & 0.81 & 2.01 & 4.22 \\
\hline & Parota & 6.36 & 5.04 & 2.67 & 15.88 \\
\hline & Rosa Morada II & 3.45 & 0.85 & 2.46 & 4.59 \\
\hline & Total & 4.31 & 3.29 & 2.01 & 15.88 \\
\hline \multirow[t]{6}{*}{ Suelo $0-15$} & Melina & 30.17 & 7.51 & 19.41 & 41.34 \\
\hline & Teca & 28.03 & 6.11 & 21.21 & 38.59 \\
\hline & Rosa Morada I & 26.94 & 11.2 & 8.59 & 41.58 \\
\hline & Parota & 37.68 & 5.42 & 29.02 & 42.93 \\
\hline & Rosa Morada II & 35.38 & 5.07 & 30.19 & 43.46 \\
\hline & Total & 31.64 & 8.09 & 8.59 & 43.46 \\
\hline \multirow[t]{6}{*}{ Suelo $15-30$} & Melina & 15.78 & 3.62 & 10.26 & 20.06 \\
\hline & Teca & 14.54 & 4.13 & 10.05 & 20.54 \\
\hline & Rosa Morada I & 21.05 & 7.41 & 15.18 & 35.05 \\
\hline & Parota & 28.27 & 5.95 & 18.51 & 35.57 \\
\hline & Rosa Morada II & 28.75 & 6.56 & 22.19 & 36.64 \\
\hline & Total & 21.68 & 8.08 & 10.05 & 36.64 \\
\hline
\end{tabular}




\begin{tabular}{cccccc}
\hline & Parcela & Media & Desviación estándar & Mínimo & Máximo \\
\hline Total & Melina & 51.73 & 12.09 & 39.35 & 71.56 \\
& Teca & 45.63 & 9.49 & 33.90 & 60.17 \\
& Rosa Morada I & 50.9 & 15.56 & 34.26 & 78.64 \\
& Parota & 73.95 & 11.3 & 55.89 & 90.78 \\
& Rosa Morada II & 69.02 & 10.48 & 57.52 & 84.17 \\
& Total & 58.24 & 15.85 & 33.9 & 90.78 \\
\hline
\end{tabular}

\section{Carbono en arbustos y herbáceas}

La concentración de carbono por los componentes de hojarasca, hierbas y arbustos fue mayor en las parcelas sin manejo debido a que no fue removido dicho componente por efectos del manejo, existiendo una diferencia aproximada de $1.5 \mathrm{Mg} \mathrm{ha}^{-1}$ en el contenido de carbono de hierbas y arbustos, mientras que, la concentración de hojarasca fue de $0.95 \mathrm{Mg} \mathrm{ha}^{-1}$ aproximadamente. Con respecto, a las parcelas sin manejo, donde se analizaron las parcelas con parota y la rosa morada II, el contenido de carbono aportado por la vegetación herbácea y arbustiva fue superior en la parota, por $0.25 \mathrm{Mg} \mathrm{ha}^{-1}$; sin embargo, no se encontraron diferencias significativas (Figura 1a).
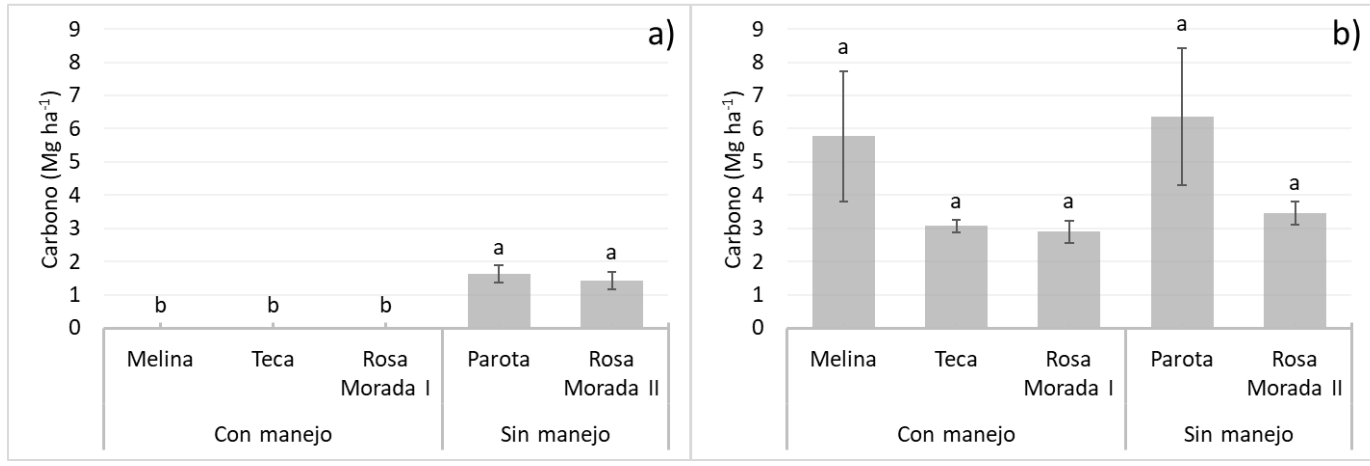

Figura 1. Medias y error estándar de cantidad de carbono $\left(\mathrm{Mg} \mathrm{ha}^{-1}\right)$ a) arbustos y hierbas; y b) hojarasca en la plantación de diferentes especies con y sin manejo. Valores promedio \pm error estándar seguidas de diferencias de letras $(\mathrm{a}, \mathrm{b})$ indican diferencia significativa para $p<0.05$.

\section{Carbono en hojarasca}

En el caso de hojarasca las parcelas con parota sin manejo fueron las que tuvieron mayor concentración de carbono $\left(6.36 \mathrm{Mg} \mathrm{ha}^{-1}\right)$, mientras que la especie que presentaron menor concentración de carbono fueron las parcelas con rosa morada I con manejo $\left(2.9 \mathrm{Mg} \mathrm{ha}^{-1}\right)$, sin que hubiera diferencia significativa en este componente, debido principalmente a su alta variabilidad en el contenido de carbono en las diferentes parcelas muestreadas (Figura 1b). En términos de estimación de biomasa en hojarasca, los valores reportados se encuentran en el rango que otros autores reportan para zonas tropicales, los cuales, varían de 3.2 a $5.2 \mathrm{Mg} \mathrm{ha}^{-1}$ de biomasa de mantillo reportados por (Klinge, 1968; Aguilar, 2004; De Miguel, 2004; Bergamini et al., 2009). 


\section{Carbono en suelo}

El mayor contenido de carbono en suelo se encontró a la profundidad de 0 a $15 \mathrm{~cm}$, en la parcela de parota con un $37.68 \mathrm{Mg} \mathrm{ha}^{-1}$ y la menor concentración se registró en las parcelas de rosa morada I con $26.94 \mathrm{Mg} \mathrm{ha}^{-1}$, encontrando diferencias significativas entre las parcelas con parota y rosa morada I, mientras que las parcelas restantes, no se encontró diferencias significativas (Figura 2a).

En la profundidad de 15 a $30 \mathrm{~cm}$, el contenido de carbono con mayor concentración fueron las parcelas de rosa morada II con un $28.75 \mathrm{Mg} \mathrm{ha}^{-1}$, muy similar a las parcelas con rosa morada II; mientras que la más baja fueron las parcelas de teca con un $14.54 \mathrm{Mg} \mathrm{ha}^{-1}$. En este componente se encuentra diferencias significativas entre las parcelas con manejo y sin manejo, mientras que entre ellas no existe diferencias (Figura 2b).

La diferencia entre estas dos profundidades refleja que en las parcelas con manejo el contenido de carbono disminuye en $50 \%$ aproximadamente, mientras que en las parcelas sin manejo solo disminuye un $30 \%$ aprox., como se puede observar en la Figura 2. Esto puede deberse a que el movimiento del humus en el perfil del suelo es descendente y su concentración disminuye conforme aumenta la profundidad del suelo (Braakhekke et al., 2013; Paz y Etchevers. 2016), pero también se puede atribuir a que en las capas de suelo superficiales se desarrolla la mayor cantidad de raíces y la actividad microbiana es mayor.
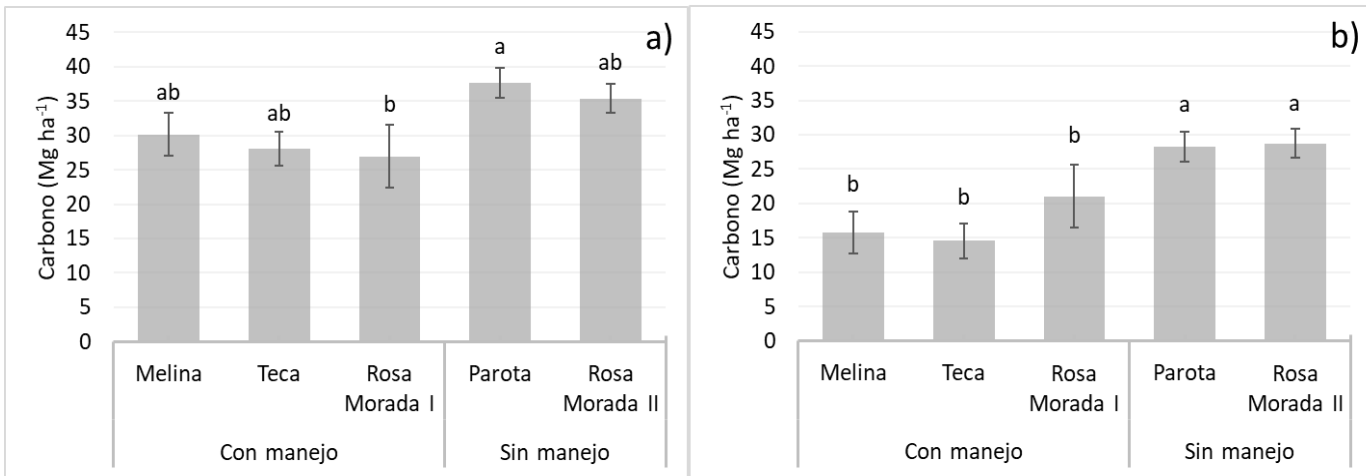

Figura 2. Medias y error estándar de cantidad de carbono en suelo ( $\left.\mathrm{Mg} \mathrm{ha}^{-1}\right)$ de dos profundidades a) 0 a $15 \mathrm{~cm}$; y b) 15 a $30 \mathrm{~cm}$ en plantación de diferentes especies con y sin manejo. Valores promedio \pm error estándar seguidas de diferencias de letras $(a, b)$ indican diferencia significativa para $p<0.05$.

Después de realizados los análisis de concentración de carbono en los suelos de las diferentes parcelas, se puede afirmar que a mayor profundidad el contenido de carbono disminuye aproximadamente 35\%. Esto se determinó después de realizar la prueba de comparaciones múltiples de Duncan, la cual evidenció que existe diferencias significativas con un $p<0.05$ entre el contenido de carbono a la profundidad de 0 a $15 \mathrm{~cm}$, comparado con la cantidad de carbono medido a la profundidad de 15 a $30 \mathrm{~cm}$ (Cuadro 2). 
Cuadro 2. Resultado de la prueba de comparaciones múltiples de Duncan entre el contenido de carbono en suelo ( $\mathrm{Mg} \mathrm{ha}^{-1}$ ) a dos profundidades en plantación de diferentes especies con y sin manejo en el Sitio Experimental Costa de Jalisco.

\begin{tabular}{|c|c|c|c|}
\hline \multirow{2}{*}{ Plantación } & \multirow{2}{*}{$\begin{array}{l}\text { Profundidad } \\
\text { (cm) }\end{array}$} & \multicolumn{2}{|l|}{ Suelo } \\
\hline & & Contenido carbono (promedio en $\mathrm{Mg} \cdot \mathrm{ha}^{-1}$ ) & Agrupación Duncan \\
\hline \multirow[t]{2}{*}{ Sin manejo } & $0-15$ & $36.53 \pm 5.15$ & $\mathrm{a}$ \\
\hline & $15-30$ & $28.52 \pm 5.98$ & $\mathrm{~b}$ \\
\hline \multirow[t]{2}{*}{ Con manejo } & $0-15$ & $28.39 \pm 8.15$ & a \\
\hline & $15-30$ & $17.12 \pm 5.79$ & $\mathrm{~b}$ \\
\hline
\end{tabular}

Además, al realizar un análisis de varianza para determinar si existen diferencias significativas entre las parcelas con manejo y sin manejo se obtuvo que la parcela con mayor contenido de carbono fue a profundidad de 0 a $15 \sin$ manejo con un $36.53 \pm 5.15 \mathrm{Mg} \mathrm{ha}^{-1}$, mientras que la menor concentración de carbono fue en las parcelas con manejo y una profundidad de 15 a $30 \mathrm{~cm}(17.12$ $\pm 5.79 \mathrm{Mg} \mathrm{ha}^{-1}$ ) (Cuadro 2). Dentro de las parcelas sin manejo a diferentes profundidades se encontró que existen diferencias significativas, al igual que entre las parcelas con manejo como se puede observar en la Figura 3.

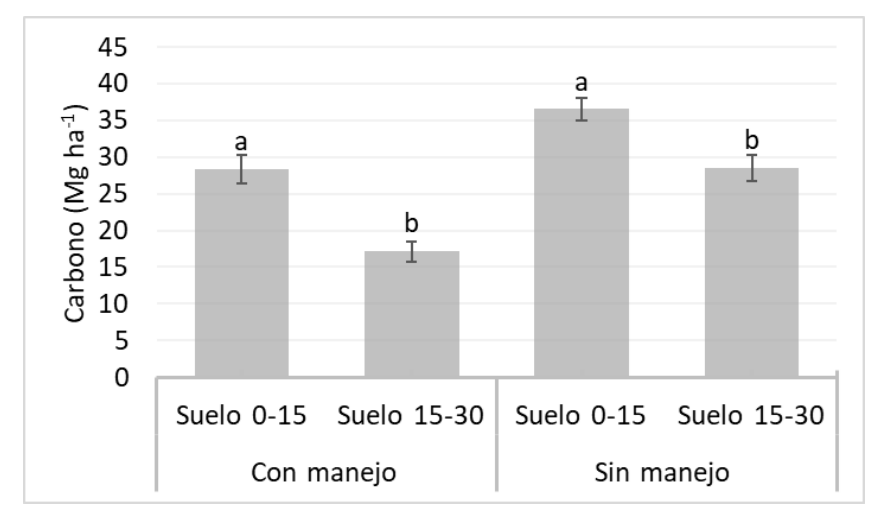

Figura 3. Cantidad de carbono en suelo $\left(\mathrm{Mg} \mathrm{ha}^{-1}\right)$ a dos profundidades a) 0 a $15 \mathrm{~cm} \mathrm{y} \mathrm{b);} 15$ a $30 \mathrm{~cm}$ en plantación de diferentes especies con y sin manejo. Valores promedio \pm error estándar seguidas de diferencias de letras $(\mathrm{a}, \mathrm{b})$ indican diferencia significativa para $p<0.05$.

Cuando se realizó la suma de los componentes (arbustivos y herbáceas, hojarasca, y suelo), se encontró que los sitios con mayor contenido de carbono fueron las parcelas sin manejo como se puede observar en la Figura 4, debido principalmente a la nula vegetación herbácea y arbustiva que se elimina en las labores culturales y mantenimiento del lugar. Además, el estudio reflejo que la concentración de carbono total, las parcelas sin manejo tuvieron 30\% mayor carbono que las parcelas con manejo, existiendo diferencia significativa. 


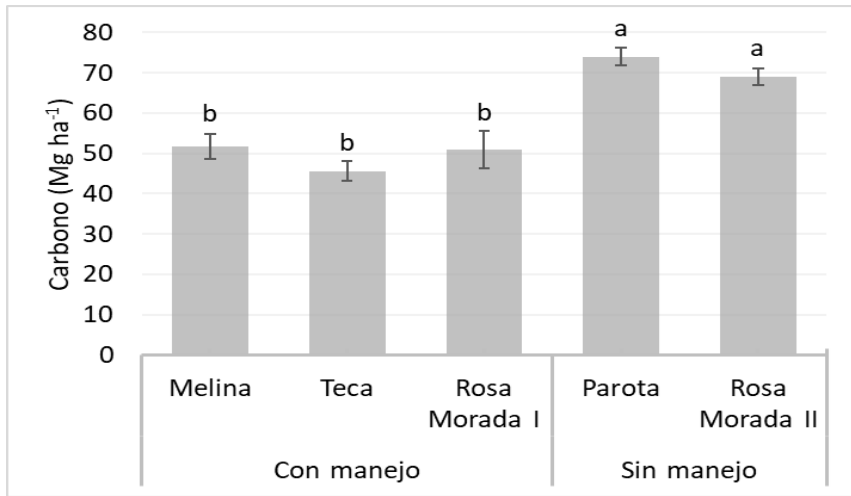

Figura 4. Medias y error estándar de cantidad de carbono total $\left(\mathrm{Mg} \mathrm{ha}^{-1}\right)$ en la plantación de diferentes especies con y sin manejo. Valores promedio \pm error estándar seguidas de diferencias de letras $(\mathrm{a}, \mathrm{b})$ indican diferencia significativa para $p<0.05$.

\section{Discusión}

El resultado obtenido en el presente trabajo pone de manifiesto que no es necesario realizar manejo, o al menos ese tipo de manejo en las parcelas con fines de captura de carbono, lo que además representaría una disminución significativa de los costos al mantener las parcelas con plantación. Finalmente, en el análisis del contenido total de carbono, se encontró que en todas las parcelas con y sin manejo es en la parte subterránea donde se encuentra el mayor contenido de carbono, siendo la parota la especie con mayor contenido de carbono con $65.95 \mathrm{Mg} \mathrm{ha}^{-1}$ y $73.94 \mathrm{Mg} \mathrm{ha}^{-1}$ si se le suma el contenido de carbono de la parte aérea; en cambio las parcelas con teca sólo alcanzaron en promedio 45.63 $\mathrm{Mg} \mathrm{ha}^{-1}$ del carbono total.

Esto podría explicarse porque el carbono en las plantas se encuentra altamente relacionado con el nitrógeno, ya que éste integra las cadenas de aminoácidos necesarias para formar las complejas proteínas que darán origen a los tejidos de las plantas. Para obtener el nitrógeno necesario del suelo, algunas plantas, como la parota, que es una leguminosa, mantienen relaciones simbiontes con microorganismos del suelo que les permiten fijar este elemento en sus raíces. Debido a este tipo de relaciones, las plantas presentan una mayor eficiencia en la realización de sus procesos metabólicos, por consiguiente, aumenta su tasa fotosintética lo cual permite una mayor acumulación de carbono en los órganos de las plantas.

De las parcelas estudiadas en el presente trabajo, rosa morada, teca, melina y parota, en el Sitio Experimental Costa de Jalisco en La Huerta, Jalisco, se puede observar que la variación en el contenido de carbono de cada una no tuvo diferencias significativas, excepto para el componente del suelo. Sin embargo, sí se observó que en las parcelas, sin manejo existe mayor concentración de carbono que aquellas con manejo; debido a que la vegetación herbácea y arbustiva se mantiene en las primeras parcelas, ya que no se extrae del ecosistema por efecto del manejo.

En cambio, en las parcelas con manejo la vegetación del sotobosque fue retirada, lo que disminuye la biomasa disponible para fijar carbono y por tanto disminuye la cantidad total de carbono presente en el sitio de muestreo. Respecto al carbono subterráneo, la mayor cantidad se encontró en las capas superficiales del suelo (de 0 a $15 \mathrm{~cm}$ ) y conforme aumentó la profundidad la concentración de carbono capturado disminuyó. 
También se encontró que el componente subterráneo es el que aporta mayor cantidad de carbono al total por parcela. Estos resultados muestran un comportamiento típico de carbono en el suelo, acordes a los estudios realizados por diferentes autores como Acosta (2003); Gómez (2008), los cuales mencionan que el contenido de carbono en los suelos disminuye conforme aumenta la profundidad.

\section{Conclusiones}

Las parcelas con mayor contenido de carbono fueron aquellas sin manejo con presencia de parota con un $73.95 \mathrm{Mg} \mathrm{ha}^{-1}$. Para la plantación con manejo se encontró una disminución del contenido del carbono en la parte aérea, principalmente proveniente de las hierbas y arbustos, debido a las labores de chaponeo de la vegetación arbustiva. La mayor concentración de carbono orgánico del suelo se encuentra en las capas superficiales $(0 \mathrm{a} 15 \mathrm{~cm})$ y conforme aumentó la profundidad, la cantidad del carbono disminuye.

\section{Literatura citada}

Acosta-M, J.; Vargas, A.; Velázquez, J. y Etchevers. 2002. Estimación de la biomasa aérea mediante el uso de relaciones alométricas en seis especies arbóreas en Oaxaca, México. Agrociencia. 36(6):725-736. http://www.redalyc.org/articulo.oa?id=30236610.

Acosta-M, M. 2003. Diseño y aplicación de un método para medir los almacenes de carbono en sistemas con vegetación forestal y agrícolas de ladera en México. Tesis de Doctorado. Colegio de Posgraduados. Montecillo, México. 135 p. http://www.sidalc.net/cgibin/wxis.exe/?IsisScript=UACHBC.xis\&method=post\&formato=2\&cantidad=1\&expresi on $=\mathrm{mfn}=099694$.

Acosta-M, M.; Carrillo-A., F. y Díaz-L, M. C. 2009. Determinación del carbono total en bosques mixtos de Pinus patula Schl. et Cham. Terra Latinoam. 27(2):105-114. http://www.scielo.org.mx/pdf/tl/v27n2/v27n2a3.pdf.

Aguilar, A. G. 2004. Los asentamientos humanos y el cambio climático. In: Martínez, J. y Fernández, A. (Comp.). Cambio climático, una visión desde México. INE-SEMARNAT. México, DF. 267-278 pp.

Bergamini, S. M.; Gattig.; Wisniewski, C.; Yukio, M. A.; Targa, C. A.; Lorenzetto, A. and Putini, F. 2009. Patterns of litter production in a secondary alluvial Atlantic Rain Forest in southern Brazil. Revista Brasil. Bot. 32(4):805-817. http://www.scielo.br/pdf/rbb/v32n4/ a18v32n4.pdf.

Braakhekke, M. C.; Wutzler, T.; Beer, C.; Kattge, J.; Schrumpf, M.; Ahrens, B.; Schöning, I.; Hoosbeek, M. R.; Kruijt, B.; P. Kabat, P. and Reichstein, M. 2013. Modeling the vertical soil organic matterprofile using Bayesian parameter estimation. Biogeosciences. 10(1):399-420. http://doi:10.5194/bg-10-399-2013.

De Jong, B. H.; Cairns, M. A.; Haggerty, P. K.; Ramirez, M. N.; Ochoa, G. S.; Mendoza, V. J.; González, E. M. and March, M. I. 1999. Land-use change and carbon flux between 1970s and 1990s in the central highlands of Chiapas, México. Environ. Manag. 23(3):373-385. https://doi.org/10.1007/s002679900193.

De Jong, B.; Montoya, G.; Nelson, G. K.; Soto-Pinto, L.; Taylor, J. and Tipper, R. 1995. Community forest management and carbon sequestration: a feasibility study from Chiapas, México. Interciencia. 20(6):409-416. 
De Miguel, S. M.; Harmand, J. M. y Hergoualc'h, K. 2004. Cuantificación del carbono almacenado en la biomasa aérea y el mantillo en sistemas agroforestales de café en el suroeste de Costa Rica. Agroforestería de las Américas 11(41-42):98-104. http://repositorio.bibliotecaorton.catie.ac.cr/bitstream/handle/11554/5997/Cuantificaci on_del_carbono_almacenado.pdf? sequence $=1 \&$ isAllowed $=y$.

FAO. 2000. Sistemas de uso de la tierra en los trópicos húmedos y la emisión y secuestro de $\mathrm{CO}_{2}$. World Soil Resources Reports 88. Rome. 98 p. http://www.fao.org/3/a-X4590s.pdf.

Figueroa, N. C. J. D.; Etchevers, B. A.; Velásquez, M. M. y Acosta, M. 2005. Concentración de carbono en diferentes tipos de vegetación de la Sierra Norte de Oaxaca. Terra Latinoam. 23(1):57-64. https://www.redalyc.org/articulo.oa?id=57323108.

García, E. 2004. Modificaciones al sistema de clasificación climática de Köppen. México. $5^{\text {ta }}$ (Ed.). Instituto de Geografía-UNAM. México, DF. 90 p. http://www.publicaciones.igg.unam.mx/ index.php/ig/catalog/view/83/82/251-1.

Gómez-D. J. D. 2008. Determinación de los almacenes de carbono en los compartimentos aéreo y subterráneo de dos tipos de vegetación en la reserva de la biósfera 'Sierra de Huautla', Morelos, México. Tesis de doctorado. Programa de Edafología. Colegio de Postgraduados. Campus Montecillo. 194 p.

IPCC. 2007. Cambio climático 2007: Informe de síntesis. Contribución de los grupos de trabajo I, II y III al cuarto informe de evaluación del grupo intergubernamental de expertos sobre el cambio climático-equipo de redacción principal: Pachauri, R. K. y Reisinger, A. (directores de la publicación). IPCC. Ginebra, Suiza. 104 p. https://www.ipcc.ch/site/assets/uploads/ 2018/02/ar4_syr_sp.pdf.

Klinge, H. 1968. Litter production in an area of Amazonian terra firme forest. Part I. Litter - fall, Organic carbon and total Nitrogen Contents of Litter). Amazoniana: Limnologia et Oecologia Regionalis Systematis Fluminis Amazonas. 1(4):287-302.

Masera, O. R.; De Jong, B. y Ricalde, I. 2000. Consolidación de la oficina mexicana para la mitigación de gases de efecto invernadero. México. 197 p.

Márquez, L. 1997. Validación de campo de los métodos del instituto Winrock para el establecimiento de pácelas permanentes de muestreo para cuantificar carbono en sistemas agroforestales. Guatemala. Universidad del Valle de Guatemala. 45 p.

Ordóñez, J. A.; De Jong, B. H. J. y Masera O. 2001. Almacenamiento de carbono en un bosque de Pinus pseudostrobus en Nuevo San Juan, Michoacán. Madera y Bosques. 7(2):27-47. https://www.redalyc.org/pdf/617/61770204.pdf.

Paz, F. y Etchevers, J. 2016. Distribución a profundidad del carbono orgánico en los suelos de México. Terra Latinoam. 34(3):339-355. https://www.terralatinoamericana.org.mx/ index.php/ terra/article/view/153/132.

Soto, P. L.; Anzueto, M.; Mendoza, J.; Jiménez, F. G. and De Jong, B. 2010. Carbon sequestration through agroforestry in indigenous communities of Chiapas, México. Agroforest Systems, 78(1):39-51. https://doi.org/10.1007/s10457-009-9247-5.

Zar, J. H. 2010. Biostatistical analysis. $5^{\text {th }}$ (Ed.). Prentice Hall. Englewood Cliffs, NJ, USA. 663 p. 